\title{
ECONOMIC EVALUATION OF A 100\% WHEY-BASED PARTIALLY HYDROLYZED FORMULA FOR THE PREVENTION OF ATOPIC DERMATITIS AMONG SWISS CHILDREN: PRELIMINARY RESULTS
}

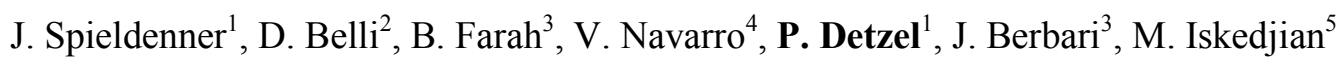 \\ ${ }^{1}$ Nestle Nutrition Institute, Vevey, ${ }^{2}$ Hôpitaux Universitaires de Genève, Geneva, Switzerland, ${ }^{3}$ PharmIdeas \\ Research and Consulting Inc, Ottawa, ON, Canada, ${ }^{4}$ PharmIdeas Europe SAS, Lyon, France, ${ }^{5}$ PharmIdeas \\ Research and Consulting Inc, Oakville, ON, Canada
}

Background and aims: An economic analysis was undertaken to determine the cost-effectiveness of a partially hydrolyzed infant formula (PHF-W) (BEBA-HA ${ }^{\circledR}$, Nestlé S.A, Switzerland) compared to standard cow's milk formula (SF) in the prevention of atopic dermatitis (AD) in at-risk Swiss children not exclusively breastfed.

Methods: The model synthesized treatment pathways (medical treatment and, in some cases, formula change), resource utilization and costs associated with the management of AD in Switzerland. The final outcome was the expected cost difference per avoided case of $\mathrm{AD}(\mathrm{ECD} / \mathrm{AC})$ for PHF-W vs. SF, presented from three perspectives: Ministry of Health $(\mathrm{MOH})$, subject's family and society (SOC). A secondary analysis compared PHF-W to extensively hydrolyzed formula (EHF) in prevention.

Results: By selecting PHF-W over SF, 1618 AD cases were expected to be avoided in a birth cohort of 22442 at-risk infants with expected additional costs of $1098 \mathrm{CHF}$ from the $\mathrm{MOH}$ perspective, and expected savings of 2067 CHF and 970 CHF from family and SOC perspectives, respectively. The main cost drivers were formula (MOH and SOC) and time loss (family). PHF-W yielded 11.1M CHF savings against EHF when EHF was assumed to be used in prevention. Sensitivity analyses confirmed the robustness of the model.

Conclusion: Under a range of assumptions, this analysis has established the dominance of PHF-W over SF from the family and SOC perspectives and attractive cost-effectiveness from the MOH perspective in the prevention of $\mathrm{AD}$ in Switzerland. Accordingly, PHF-W should be more widely adopted, and its reimbursement should be considered in at-risk infants. 